\title{
Immunoglobulin treatment in human and experimental epilepsy
}

\author{
Baziel G M van Engelen, Willy O Renier, Corry M R Weemaes
}

\begin{abstract}
The relationship between human immunoglobulin administration, and human and experimental epilepsy was investigated by reviewing the literature as well as the authors' clinical and experimental studies. The focus was: 1 ) the possible efficacy of IVIg; 2) the possibility that IVIg significantly increases CSF IgG and could reach the brain, and 3 ) the interaction between the IVIg preparation and epilepsy. There is still no formal proof of efficacy of IVIg treatment in epilepsy. However, the clinical data presented suggests that IVIg is likely to be effective in a subgroup of patients with intractable and epilepsy, and may be considered as a safe add-on medication in various types of idiopathic and symptomatic intractable epilepsy. From the authors' study on cerebrospinal fluid IgG concentrations before and after IVIg treatment in patients with epilepsy, it is concluded that the main component of the IVIg preparation (the IgG molecule) crosses the blood-CSF barrier, significantly increases CSF IgG concentration, and may reach the brain and act centrally. From a review of previous reports and two immunological studies carried out by the authors it is concluded that immunogenetic mechanisms may play a role in triggering or maintaining some types of epilepsy. In view of this, IVIg could act on epilepsy by its broad immunomodulatory mechanism of action. In addition, preliminary data are reported that contribute to the hypothesis that IVIg may have a direct neuromodulating effect.
\end{abstract}

Neuroimmunology is one of the attempts to bridge the gap arising from compartmentalisation. In general, there is no doubt concerning a bidirectional interaction between the nervous and the immune system. ${ }^{1}$ Nevertheless, since an empirical start in $1977,{ }^{2}$ it remains controversial whether there is such an interaction between the nervous system and the immune system with respect to immunoglobulin administration in epilepsy. In this paper we investigate this interaction. The major questions are: 1) What data support the possible efficacy of immunoglobulin in epilepsy? We address this question by reviewing previous studies and reporting our own pilot study on intravenous immunoglobulin (IVIg) in 15 patients with intractable childhood epilepsy; 2) Does IVIg significantly increase cerebrospinal fluid (CSF) IgG and could it reach and interact with the brain? We therefore performed CSF investigations before and after IVIg treatment; 3) If IVIg seems effective in some patients with epilepsy, what could be the mechanism of action? Are there indications for an immunological mechanism in epilepsy (we report two of our immunological studies and review other immunological studies on epilepsy), or does IVIg act directly as an anticonvulsant by a neuromodulating mechanism? We also discuss preliminary data on the effect of human IVIg administration in experimental epilepsy.

\section{1) Possible efficacy of IVIg in intractable epilepsy}

To ascertain possible efficacy, we identified all articles on IVIg (for reasons of convenience the abbreviation IVIg will be used for immunoglobulin administered intravenously and intramuscularly) treatment in intractable epilepsy by performing an English, German, and French-language literature search using MEDLINE (1966-93), by reviewing meeting reports, and by extensive hand search of bibliographies. In 24 studies, none with a placebo controlled design, 368 patients with epilepsy who were receiving IVIg were identified. ${ }^{3}$ Patients' ages ranged from $<1$ to 35 years, mean $7 \cdot 3$ years. Female/male ratio was $0 \cdot 6$. All patients had intractable epilepsy. On average the percentage of patients with an $\operatorname{IgG}_{2}$ deficiency was $25 \%$. The total dose of IVIg varied between 0.3 and $6.8 \mathrm{gm} / \mathrm{kg}$ for a period of 0.15 to 12 months. Whenever reported, adverse effects of IVIg were minimal. None of the studies reported the need to stop IVIg administration because of adverse effects. On average the mean percentage of clinical seizure reduction, and the mean percentage of electroencephalographic (EEG) improvement was $52 \%$ and $45 \%$, respectively. The average percentage of patients with complete seizure remission, and of patients with behavioural improvement was $23 \%$ and $63 \%$, respectively.

Cumulative meta-analysis of the reports was not possible because of the lack of controlled studies, the heterogeneity of the available studies, and the possible publication bias of unpublished negative data. Because of these pitfalls, we carried out our own pilot study to find out if it merited a more extended and controlled trial. Our pilot study also lacks a randomised and controlled 
design, but partly corrects for this by "blind" scoring of the EEGs before and after treatment.

In an add-on pilot study, ${ }^{4}$ a group of 15 children with cryptogenic and intractable West syndrome (WS, $n=3$ ) and LennoxGastaut syndrome (LGS, $n=12$ ) received IVIg $(0.4 \mathrm{~g} / \mathrm{kg}$ body weight per day for five consecutive days, followed by the same dose once every two weeks for three months). IVIg was produced from the plasma of more than 3000 Dutch blood donors by ethanol fractionation, treated at $\mathrm{pH} 4$ with a low concentration of pepsin (Central Laboratory of the Netherlands Red Cross Blood Transfusion Service, Amsterdam, NL). Five patients had been treated previously with adrenocorticotrophic hormone (ACTH) without success. The reduction in clinical seizures averaged $70 \%$. One patient showed total cessation of seizures. EEG recordings, scored "blind", revealed a mean reduction in spike wave discharges of $40 \%$. In all 15 patients acceleration of EEG background activity occurred, and psychomotor development (measured by the Denver Developmental Screening Test) improved.

The favourable results of this study agree with the data derived from review of the literature. ${ }^{3}$ Although there is still no formal proof of efficacy of IVIg treatment in epilepsy, the clinical data suggest that IVIg is likely to be effective in some patients with intractable epilepsy, and may be considered as a safe add-on medication in intractable epilepsy. Intractability is defined as insufficient control of epilepsy by adequate doses of antiepileptic drugs.

\section{2) CSF investigations in intractable} epilepsy before and after IVIg treatment Before and after IVIg administration, the CSF was examined ${ }^{5}$ in the group of 15 patients with cryptogenic types of WS and LGS. Compared with an age-matched control group, no CSF abnormalities were found before or after IVIg administration. This agrees with the concept of cryptogenic aetiology. Hypotheses concerning seizure consequences, such as, increased blood-CSF permeability, increased markers of brain cell destruction, or increased metabolic components could not be confirmed.

After IVIg administration in these patients with WS or LGS (all with a generally undisturbed blood-CSF barrier permeability as determined by $Q$ albumin $=C S F$ albumin concentration $\times 10^{5} /$ serum albumin concentration) CSF IgG concentrations increased significantly and proportionally to the $Q$ albumin level. The serum total IgG concentration and the CSF IgG concentration increased by an average of $76 \%$ and $44 \%$, respectively. There were no signs of adverse effects (such as, aseptic meningoencephalitis) after 165 infusions of IVIg performed on the 15 children.

From this study we conclude that the main component of the IVIg preparation (the IgG molecule) crosses the blood-CSF barrier, significantly increases the CSF IgG concentration, and is likely to reach the brain and act centrally.

\section{3) The interaction between IVIg and epilepsy}

IVIg treatment in epilepsy started empirically. ${ }^{2}$ Now we need to make a more rational study of the possible mechanisms of action of IVIg in intractable epilepsy. We have attempted to bridge the gap between intractable epilepsy and the broad immunomodulatory effect of IVIg treatment, by discussing: A) indications for an immunological mechanism in epilepsy, and B) indications of a possible non-immunological mechanism of action of IVIg.

\section{A) AN IMMUNOLOGICAL MECHANISM IN} EPILEPSY (CLINICAL RESEARCH)

The hypothesis that immunological mechanisms are involved in the pathogenesis of epilepsy is not new. The old allergic approach to epilepsy ${ }^{67}$ has been revived and transformed to an immunological one based on additional clinical and experimental findings. For a review of the experimental findings we refer to Aarli. ${ }^{8}$

We have focussed on the clinical investigations but immunological data in the various studies are difficult to compare, and the contribution of anticonvulsant drugs to the immunological abnormalities is not always clear. Reported laboratory investigations in favour of an immunological aspect in human epilepsy include: an impaired humoral immunity predominantly $\operatorname{IgA}$ and $\operatorname{IgG}_{2}$ deficiencies, ${ }^{9-12}$ lower kappa/lambda ratios of serum IgG, and serum IgM, ${ }^{13}$ an increased incidence of antibodies against the frontal cortex, ${ }^{14}{ }_{15}$ increased percentage of B-cells, ${ }^{16}$ decreased percentage of $\mathrm{T}$-cells, ${ }^{12}$ decreased CD4-CD8 $\mathrm{T}$-cell ratios, ${ }^{17}$ and differences in HLA class II antigen frequencies compared with the general population. ${ }^{18-21}$ The IgA deficiency found in $5-10 \%$ of patients with epilepsy is usually drug-induced, but such patients can have subnormal IgA before treatment. ${ }^{8}$

Other indications in favour of an immunological involvement in epilepsy are the clinical experience of exacerbation but also of total remission of intractable childhood epilepsy after viral infections. ${ }^{22}{ }^{23}$ Indirect evidence for reciprocal interactions between the immune and nervous system in epilepsy are the immunological alterations induced by anticonvulsant drugs such as, phenytoin, and carbamazepine, ${ }^{84}$ and the anticonvulsant effect of immunosuppressive drugs such as ACTH and corticosteroids in otherwise intractable childhood epilepsy like West syndrome. ${ }^{26}$

In conclusion, there is evidence for an association between epilepsy and immunological disturbances in some patients. This association, however, has not yet been firmly explained and the most fundamental questions concerning this association remain 
unsolved. Firstly, immunological factors may contribute to the development of epilepsy. Secondly, it is possible that as a result of seizures, CNS antigens may become exposed to and trigger the immune system. Thirdly, another possibility is misregulation of the immune system in epilepsy, due to a common underlying biological process that during development concurrently gives rise to immune misregulation and to CNS disturbances leading to epilepsy. ${ }^{14}$

Our own immunological studies in twelve children with LGS involved a functional test of overall immune responsiveness (in vivo primary humoral immune response against the neoantigen haemocyanin), and serological HLA typing for class I as well as class II antigens.

The in vivo primary humoral immune response against haemocyanin was determined, in addition to measurements of serum total IgG, IgA and IgM concentrations, IgG subclass concentrations, percentages of Bcells, percentages of CD3, CD4, and CD8 positive T-cells, and in vitro lymphocyte proliferation. Total IgG concentrations were significantly increased in patients with LGS compared with the normal control group ( $\mathrm{p}<$ $0 \cdot 05)$. The peak IgM response after two weeks of immunisation with haemocyanin was decreased in the LGS group compared with the epilepsy control group (on the same anticonvulsant medication, $p<0.05)$ and to the normal controls $(p<0.005)$. The IgA response after two weeks was reduced in both the LGS and the epilepsy control group compared with normal controls (both $\mathrm{p}<0.05$ ). The IgG response did not differ significantly between the three groups $(p>0.05)$, although there was a trend towards lower IgG responses in the LGS group compared with normal controls $(p=0.04)$.

The combination of immunological findings in the patients with LGS, can be considered as the expression of a dysbalanced-triggered (elevated IgG concentrations) as well as functionally impaired (decreased humoral immune response to a primary antigen)-immune system. This combination of findings has also been described in an autoimmune disease like systemic lupus erythematosus in humans and in epilepsy-prone rats.

Serological HLA class I and class II typing was performed on 12 patients with cryptogenic LGS and compared with a normal control group of 1661 Whites. ${ }^{21}$ In the LGS group we found a significant increase in the frequency of DR5 antigen (55\%, Chi-square $=5 \cdot 6)$, and an indication of a decrease in the frequency of DR4 antigen $(0 \%$, Chi-square = $3.0)$ compared with controls $(20 \%$ and $28 \%$, respectively). No significant differences existed in the frequencies of HLA class I antigens (HLA-A, B, and C) between the LGS group and the controls. Because HLA class II antigens are crucial for the immune response, the HLA class II antigen associations (that is, an increase in the frequency of DR5 antigen and an indication of a decrease in the frequency of DR4 antigen) could make individuals susceptible to developing epilepsy when exposed to various factors such as viral infections, and could be compatible with an (auto)immune genesis of these epilepsies.

These findings contribute to the hypothesis that immunogenetic mechanisms may play a role in triggering or maintaining cryptogenic LGS. IVIg could thus act on epilepsy by its broad immunomodulatory mechanism of action, for example, by restoration of a normal immune repertoire or by anti-idiotype interaction. ${ }^{26}$

\section{B) A NEUROMODULATING OR DIRECT ANTICONVULSANT EFFECT OF IVIg (EXPERIMENTAL RESEARCH)}

In humans, IVIg has been reported to be beneficial for more than 35 diseases that may be produced by immunopathology. ${ }^{26}$ However, its exact mechanisms of action remain largely unknown, especially in neurological diseases, where it is undetermined whether IVIg in addition to its accepted immunological mechanism of action may also exhibit a direct neuromodulating effect. There are some indications which may support a nonimmunological mechanism of action of IVIg in epilepsy: 1) an immediate clinical treatment effect in some patients with otherwise intractable epilepsy, that is present not in a single type but in various types of idiopathic and symptomatic epilepsy suggesting direct interference with the final common pathway of brain disturbance; and 2) an anticonvulsant effect in an animal model of kindled cats. ${ }^{27}$ Because the possibility of a direct neuromodulating effect has been denied without (published) data, ${ }^{28}$ we investigated the anticonvulsant effect of IVIg in a direct cortical stimulation model of epilepsy. ${ }^{29}$

A total of 55 female Wistar rats was used. Human IVIg (Central Laboratory of the Netherlands Red Cross Blood Transfusion Service, Amsterdam, NL) was administered in a comparable dose as in humans (400-1500 mg/kg bodyweight) by a single intraperitoneally (ip) administered dose. In one control experiment human albumin (400-1000 mg/kg bodyweight ip) was used. Overall, six $(11 \%)$ of 55 animals receiving IVIg showed a significant increase in seizure threshold, starting within the first hour after administration and continuing for at least 40 hours. This effect of IVIg was remarkably variable between and even within the various experiments. The only other study reporting the administration of human IVIg in an animal model of epilepsy (amygdaloid kindled cats) found a comparable time-effect relationship, ${ }^{27}$ but the variability in IVIg effect was found to a lesser degree. In that study eight of 10 animals showed a favourable response. Both studies found a similar immunohistochemical staining pattern for human IgG. IgG penetrated the blood-brain barrier and could be found around blood vessels. It was even taken up by some nerve and glial cells in the cerebral cortex and deep structures of the central nervous system. 
In conclusion, IVIg shows a direct anticonvulsant effect in a subgroup of tested animals. This effect could be mediated by its main component, the IgG molecules, because they reached the brain and could be found around blood vessels and even in some neural and glial cells. IgG Fc receptors have been described in human and rat brains. ${ }^{30}$ Nevertheless, we cannot exclude an effect of cytokines or other humoral factors which are induced by, or may be present in low concentrations in IVIg preparations and are known to have an effect on CNS functioning and EEG. ${ }^{31}$ The results in an animal model, confirm the clinical impression of the variability of the IVIg effect, and the existence of a subgroup showing a favourable response.

\section{4) Conclusion}

The studies discussed indicate that there may be a bidirectional interaction between the immune system and nervous system ${ }^{1}$ for IVIg treatment of epilepsy. Firstly, some immune abnormalities are found in some types of human epilepsy, and secondly, IVIg causes an anticonvulsant effect in a subgroup of animals, whereas an effect on human epilepsy is likely.

IVIg treatment offers the possibility of approaching the nervous system via the immune system. It represents a stage in the search for more specific neuromodulating immune factors. If an anticonvulsant effect can result from application of such relatively crude IVIg preparations, appropriately targeted reagents may have clearly improved efficacy.

1 Reichlin S. Neuroendocrine-immune interactions. $N$ Engl f Med 1993;329:1246-53

2 Péchadre JC, Sauvezie B, Osier C, Gibert J. Traitement des encéphalopathies épileptiques de l'enfant par les gammaglobulines. Rev EEG Neurophysiol 1977;7:443-7.

3 van Engelen BGM, Renier WO, Weemaes CMR Gabreels FJM, Meinardi H. Immunoglobulin treatment in epilepsy, a review of the literature. Epilepsy Res (in

4 Van Engelen BGM, Renier WO, Weemaes CMR, Strengers PFW, Bernsen PJH, Notermans SLH. Highdose intravenous immunoglobulin treatment in cryptogenic West and Lennox-Gastaut syndrome; an add-on study. Eur $\mathcal{F}$ Pediatr (in press).

5 van Engelen BGM, Renier WO, Weemaes CMR, Lamers KJB, Gabreels FJM, Meinardi H. Cerebrospinal fluid examinations in cryptogenic West and Lennox-Gastaut syndrome before and after intravenous immunoglobulin syndrome before and after intravenous immu
administration. Epilepsy Res 1994;10:139-47.
6 Dewar DC. The allergic approach to epilepsy: a critical review. The Practitioner 1941;147:776-88.

7 Walker AE. Allergic phenomena as basic mechanism in epilepsy. In: Jaspar HH, Ward AA Jr, Pope A, eds. Basic mechanisms of the epilepsies. London: Churchill 1969: 812 .

8 Aarli JA. Epilepsy and the immune system. In: Aarli JA Behan WMH, Behan PO, eds. Clinical neuroimmunology. Blackwell: Oxford, 1987:385-403.

9 Baetz-Greenwalt B, Rothner AD, Saracusa C, Erenberg $G$, Cohen B, Cruse R. Lack of benefit of intravenous gamma globulin in treatment of intractable childhood epilepsy in children with and without IgG-subclass deficiency. Ann Neurol 1992;32:434.

10 Duse $\mathrm{M}$, Tiberti A, Plebani A, et al. $\mathrm{IgG}_{2}$ deficiency and intractable epilepsy of childhood. Monogr Allergy 1986;20:128-34.

11 Fontana A, Grob PJ, Sauter R, Joller H. IgA deficiency, epilepsy and hydantoin medication. Lancet 1976;1: 228-31.

12 Montelli TCB, Mota NGS, Peracoli MTS, et al. Immunological disturbance in West and LennoxGastaut syndromes. Arq Neuropsiquiatr 1984;42:132-9.

13 Haraldsson A, van Engelen BGM, Renier WO, Bakkeren JAJM, Weemaes CMR. Light chain ratios and concentrations of serum immunoglobulins in children with epilepsy. Epilepsy Res 1992;13:255-60.

14 Pliopys AV, Greaves A, Yoshida W. Anti-CNS antibodies in childhood neurologic diseases. Neuropediatrics 1989 in childhood

15 Mota NGS, Rezkallah-Iwasso MT, Peracoli MTS, Montelli TCB. Arq Neuropsiquiatr 1984;42:126-31.

16 Hrachovy RA, Frost JD, Shearer WT, et al. Immunological evaluation of patients with infantile spasms. Ann Neurol 1985;18:414.

17 Eeg-Olofsson O, Osterland CK, Guttmann RD, et al. Immunological studies in focal epilepsy. Acta Neurol Scand 1988;78:358-68.

18 Smeraldi A, Scorza Smeraldi R, Cazzullo CL, Guareschi Cazzullo A, Fabio G, Canger R. Immunogenetics of the Lennox-Gastaut syndrome: frequency of HL-A antigens Lennox-Gastaut syndrome: frequency of HL-A antigens
and haplotypes in patients and first-degree relatives. and haplotypes in patients

19 Minev M, Martinova F, Belopitova L. On the association of the HLA system with epilepsy in children. Epilepsia 1987;28:74-6.

20 Hrachovy RA, Frost JD, Pollack MS, Glaze DG Serologic HLA typing in infantile spasms. Epilepsia 1988;29:817-9.

21 van Engelen BGM, de Waal LP, Weemaes CMR, Renie WO. Serologic HLA Typing in cryptogenic LennoxGastaut syndrome. Epilepsy Res 1994;17:43-7.

22 Lombroso CT. A prospective study of infantile spasms: clinical and therapeutic correlations. Epilepsia 1983;24: 135-58.

23 Terada $M$, Kudo $H$, Abe $T$, Naito $H$, Nihei $K$ Unexpected improvement of intractable epilepsy following infections. Brain Dev 1984;6:422.

24 Pacifi $R$, Paris L, Di Carlo S, Pichini S, Zuccaro P. Immunologic aspects of carbamazepine treatment in Immunologic aspects of carbamazepine

25 Hrachovy RA, Frost JD. Infantile spasms. Pediatr Clin $N$ Am 1989;36:311-29.

26 Dwyer JM. Manipulating the immune system with immune globulin. $N$ Engl f Med 1992;326:107-16.

27 Hirayama H, Kurimoto T, Wada S, et al. Antiepileptic effects of globulin- $\mathbf{N}$, an intact human immunoglobulin and its tissue-distribution in kindled cats. Int $\mathcal{f}$ Clin Pharm 1986;2:93-7.

28 ASHP Commission on Therapeutics. ASHP therapeutic guidelines for intravenous immune globulin. Clin Pharmacy 1992;11:117-36.

29 Voskuyl RA, Dingemanse J, Danhof M. Determination of the threshold for convulsions by direct cortical stimulation. Epilepsy Res 1989;3:120-9.

30 Peress NS, Siegelman J, Fleit HB. High avidity periventricular IgG-Fc receptor activity in human and rabbit brain. Clin Immunol Immunopathol 1987;42:229-38.

31 Korneva EA. Electrophysiological analysis of brain reaction to antigen. Ann NY Acad Sci 1987;296:318-37. 